\title{
Modelling the Io-related DAM emission by modifying the beaming angle
}

\author{
L. C. Ray ${ }^{1}$ and S. Hess ${ }^{1,2}$
}

Received 7 August 2008; revised 23 September 2008; accepted 30 September 2008; published 22 November 2008.

[1] The Io-Jupiter interaction generates strong decametric radio emissions (DAM), which appear as arcs in the time-frequency plane. These emissions are beamed at an angle from the magnetic field lines, which may vary with frequency and longitude amongst other properties. Empirical models of this beaming angle describe the shape of the DAM arcs and offer insight into the emission mechanism for DAM. Several studies have investigated the variation in the emission beaming angle. The studies span a range of frequencies which depend on the observational means (spacecraft, ground-based radio telescopes) used to obtain data. Subsequently, because of the varying assumptions made (e.g. relativistic vs. non-relativistic electrons for the wave polarization), methods used (e.g. prescribing a beaming angle function vs. determining a beaming angle function from observational geometry) and frequency ranges observed, different results have been found in each study. In the present paper, we model the shape of the emission with an empirical beaming angle function and adjust the parameters to best fit the emission arcs. However, our model builds on previous models by taking into account the location of Io in the Jovian magnetic field. We also look at a broader frequency range than many of the intermediate studies. We find that a simple empirical beaming angle function describes the shape of the $\mathrm{A}, \mathrm{B}$, and $\mathrm{D} \operatorname{arcs}$ and that the beaming angle function must decrease at high and low frequencies. We then propose a simple explanation for the beaming angle profile, deduced from cyclotron maser theory.

Citation: Ray, L. C., and S. Hess (2008), Modelling the Io-related DAM emission by modifying the beaming angle, J. Geophys. Res., 113, A11218, doi:10.1029/2008JA013669.

\section{Introduction}

[2] The discovery of Jovian decametric (DAM) radiation was purely accidental. While observing the Crab nebula, Burke and Franklin [1955] detected an unknown radio source at $22.2 \mathrm{MHz}$. The source appeared for 3 months at approximately the same position, but shifted in right ascension. This shift eliminated the possibility of astronomical origin and the localized sidereal time of the emissions discounted terrestrial sources. Hence Burke and Franklin [1955] compared the source location with the positions of solar system bodies and concluded that the radio emission was associated with Jupiter. The connection between the DAM and Io was discovered by Bigg [1964], who noted a pattern when the emissions were organized by Io phase.

[3] Io, Jupiter's nearest Galilean moon, loses neutral mass to the Jovian system at a rate of $\sim 1000 \mathrm{~kg} / \mathrm{s}$. The neutral particles are quickly ionized by processes such as electron

\footnotetext{
${ }^{1}$ Laboratory for Atmospheric and Space Physics, University of Colorado, Boulder, Colorado, USA.

${ }^{2}$ LUTH, Observatoire de Paris, CNRS, Université Paris Diderot, Meudon, France.

Copyright 2008 by the American Geophysical Union. 0148-0227/08/2008JA013669
}

impact ionization, charge-exchange and photo-ionization (see review by Thomas et al. [2004]) creating a plasma torus around Jupiter at Io's orbital radius. The new plasma initially has the same orbital velocity as Io, which is approximately four times less than that of Jupiter, but is soon accelerated to corotation with Jupiter's magnetic field. The time delay of the acceleration toward corotation with Jupiter results in a wake downstream of Io. In Io's reference frame, the motion of Jupiter's magnetic field, and hence the frozen-in plasma torus, generates a corotational electric field and Alfvén waves that accelerate electrons and ultimately cause aurorae and decametric radio emissions above Jupiter's atmosphere (see reviews by Zarka [1998], Clarke et al. [2004], Saur et al. [2004]).

[4] The Io-related DAM is categorized into four source regions in Io-phase - Central Meridian Longitude (CML) space: A, B, C, and D (Figure 1). Figure 2a shows the position of the source regions relative to Jupiter in the reference frame of the observer. This can also be expressed in terms of the emission hemisphere and Io's phase, $\Phi_{I o}$, which is the angle between Io and the observer's antimeridian, as shown in Figure 2b. Each source is related to a particular shape of arc described by the arc vertex. The arc vertex occurs where the direction of the drift in emission frequency changes. Vertex-early events occur for $\Phi_{I o}<180^{\circ}$ and appear as "open parentheses", while vertex-late events 


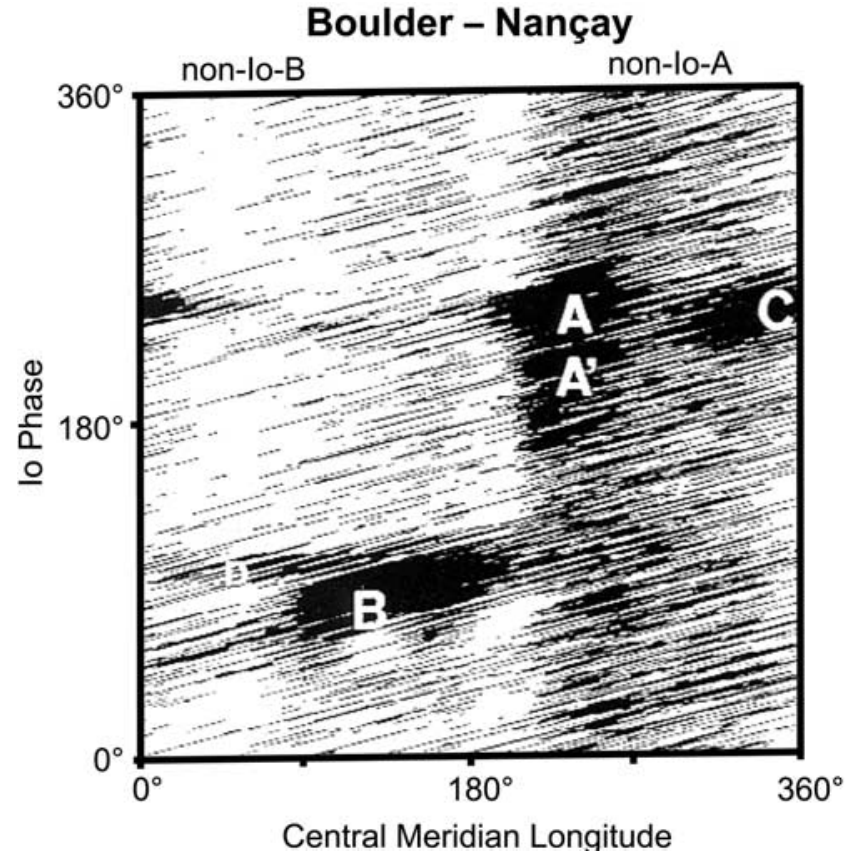

Figure 1. Source regions in Io phase-CML space. There are 4 distinct Io source locations. The background emission is thought to be associated with a separate emission mechanism.

occur for $\Phi_{I o}>180^{\circ}$ and appear as "closed parentheses". In the northern hemisphere, the early and late source regions are " $\mathrm{B}$ " and " $\mathrm{A}$ " respectively, and in the southern hemisphere "D" and "C".
[5] Moreover, the emissions occur for emitting field lines across a given range of jovian longitude and thus the visibility of the arcs, such as those in all panels of Figure 7, depends on both the phase and System III longitude of Io $\left(\lambda_{I I I}^{I o}\right)$ relative to the observer longitude $\left(\lambda_{I I I}^{o b s}\right)$, also called the central meridian longitude (CML) (Figure 2b). Because of the Jovian field orientation, A and B emissions are typically right-hand $(\mathrm{RH})$ polarized, while $\mathrm{C}$ and $\mathrm{D}$ emissions are mostly left-handed (LH). The wake preceeding Io (Figure 2b) may cause observed secondary DAM arcs, such as those in Figure $7 b$.

[6] The most commonly cited emission mechanism for DAM, as for most planetary auroral radio emissions, is the cyclotron maser instability (CMI) (see review by Zarka [2000]). This exists when there is a resonance between the gyration of the electrons around the magnetic field lines as defined by the electron cyclotron frequency $\left(\Omega_{c}\right)$ and a right-handed circularly polarized wave with a frequency near the local electron cyclotron frequency. This instability requires that the electron distribution has a positive gradient along the perpendicular velocity, $\partial F / \partial v_{\perp}>0$, which can be created by a magnetically mirrored population of electrons presenting a "loss-cone" because of the loss of particles by collision in the jovian ionosphere, a ring beam distribution, or by electron beams accelerated by the Io-Jupiter interaction. The CMI generates emission that has a narrow range of beaming angle which is symmetric relative to the magnetic field lines (i.e. the waves are emitted along a hollow cone). A more detailed description of the CMI is presented in section 4.3.

[7] The main theory regarding the jovian radio arc morphology in the time-frequency plane is that the arcs a)

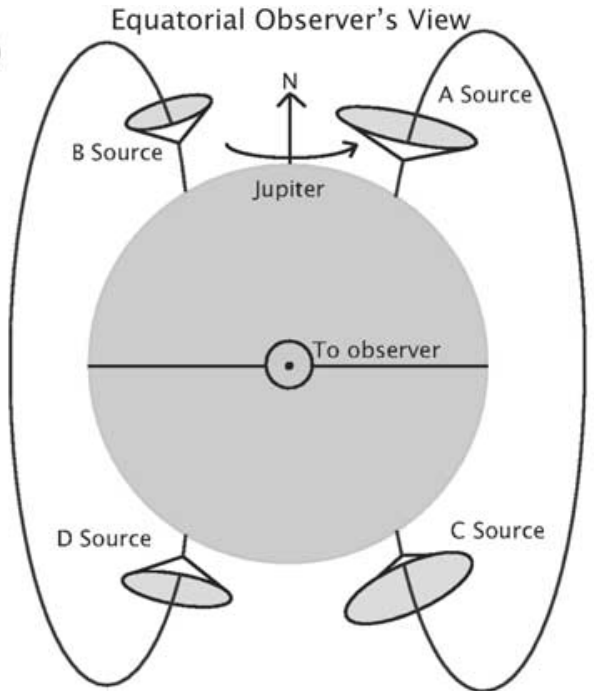

b)

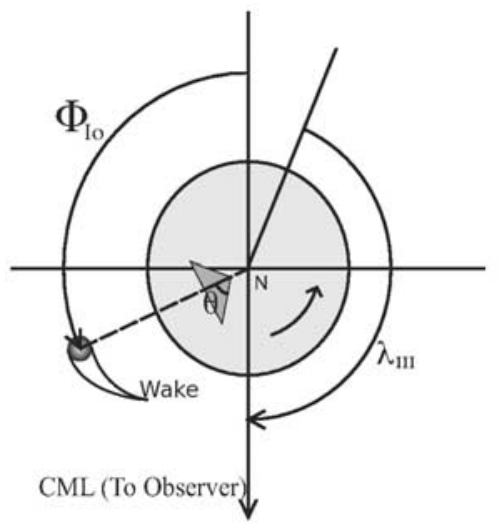

c)

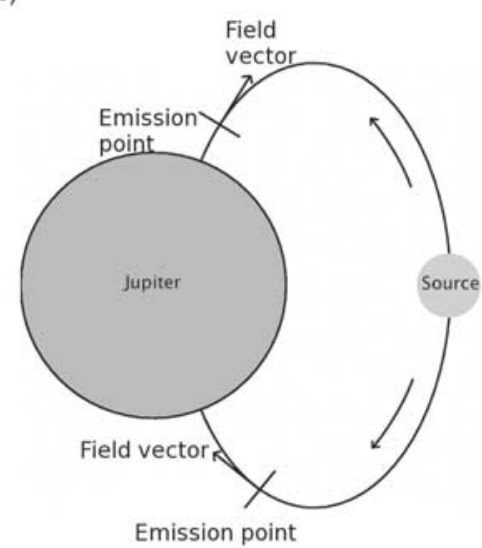

Figure 2. (a) DAM source regions. Radiation is beamed in a hollow cone. Sources A and B emit from the Northern hemisphere; C, D emit from the Southern hemisphere. B and D are early sources, that is, the observers sees the emission from the leading edge of the cone. Conversely, A and C are late sources where the emission observed is from the trailing edge of the cone. (b) Top down view of the Jupiter-Io system. The Io phase is a right-handed coordinate system initialized at the observer's anti-meridian, whereas the longitude System III $\lambda_{I I I}$ is a left-handed system fixed by the magnetic field. Thus the longitude of Io is given by $\Phi_{I o}=\lambda_{I I I}^{o b s}-\lambda_{I I}^{I O}+180^{\circ}$. (c) The radiation is beamed along an hollow cone with a beaming angle $\Theta$. When the border of the cone is aligned with the observer longitude (CML) the emission can be observed. 
are a geometrical effect because of the beaming anisotropy and the variation of the angle of observation with Jupiter's rotation. The most variable and least determined parameter in modelling the Io-related arcs is the beaming angle function, $\Theta(f)$, where $f$ is the emission frequency. This function dictates whether or not the observer will detect emission and if so, the shape of the observed arcs. Past studies have attempted to determine $\Theta(f)$, each using a different method. Using Voyager data and an assumed field geometry, Goldstein and Thieman [1981] found the angle between the observer and local field vector at the emission point for an emitting field line fixed in Jupiter's reference frame. They then applied and adjusted an empirical beaming angle function. The beaming function depended only on frequency and did not vary with source region, hence their analysis was independent of Io's location and decoupled the emissions from the Io-Jupiter interaction.

[8] Menietti et al. [1984] used a three-dimensional ray tracing code to determine the beaming angle of the emission while taking into account the Doppler shift of the frequency at the source point. Their analysis looked at selected emission frequencies and found a beaming angle which had a peak at $\sim 10 \mathrm{MHz}$ and decreased on either side. Their results were independent of the emission mechanism.

[9] Leblanc et al. [1994] and Willes et al. [1994] used the polarization of the emission to determine the beaming angle. The Leblanc et al. [1994] analysis assumed non-relativistic electrons and related the polarization of the emission directly to the beaming angle at the source, while fixing the field line in Io's reference frame. They determined the beaming angle, independent of frequency, for 33 Io-controlled events, and showed that the beaming angle varies with the system III longitude of Io $\left(\lambda_{I I I}^{I o}\right)$ and the hemisphere of emission. Willes et al. [1994] investigated the polarization of the emission in the mildly relativistic limit. The assumption of mildly relativistic electrons is consistent with the elliptical polarization of the DAM, however it predicted a beaming angle where $\Theta \lesssim 50^{\circ}$. This beaming angle was less than that found by Leblanc et al. [1994] and inconsistent with the viewing geometry. The discrepancy between the predicted angle and the viewing geometry was postulated to be due to the magnetic field model.

[10] Lecacheux et al. [1998] showed that the beaming function cannot be constant along the field lines, specifically it must decrease at the highest and lowest frequencies. They proposed refraction at the source region in Jupiter's ionosphere at high frequencies and possible refraction through the plasma torus at low frequencies to explain the decrease. Queinnec and Zarka [1998] worked backward to derive $\Theta(f)$ given the location of the observer, emission point, and Io in the jovian magnetic field using Earth-based observations from the Nançay decameter array and WIND spacecraft which spanned a large-frequency range. They measured a beaming angle function which varies with the frequency, hemisphere, and $\lambda_{I I I}^{I o}$. In particular they observed a decrease of the beaming angle at high frequency. In their model the emitting field line is fixed in Io's frame.

[11] The aforementioned investigations did not study the variation of the beaming angle at low frequency, with the exception of Goldstein and Thieman [1981] which did not include possible variations with $\lambda_{I I I}^{I O}$ and did not allow the emitting field line to shift in the Io-Jupiter interaction. Thus we use a modified form of the beaming angle function used in Goldstein and Thieman [1981] and adjust the parameters to fit DAM events from all source regions. We compare the results amongst source regions.

\section{Model}

[12] Our model computes the shape of the emission in the time-frequency plane and compares it with that observed by the planetary radio astronomy (PRA) instruments on Voyager 1 and 2. The observations were made in March (Voyager 1) and July (Voyager 2) 1979 as each spacecraft passed Jupiter. Since the PRA instrumentation was identical between the two spacecraft, we do not note from which spacecraft each observation is from. A detailed review of the observed radio emissions can be found in Boischot et al. [1981].

[13] The geometry of our model is shown in Figure 2c. First, at each point along the emitting magnetic field line, we compute the angle between the magnetic field and the observer's line of sight. We choose to simulate the emission using the instantaneous Io field line. However a field line from the Io wake is also tried with a lead angle of $15^{\circ}$ for this analysis [Clarke et al., 1998], corresponding to the difference between the FUV observations of the Io footprint and the predictions of the location of the Io footprint from the VIP4 field model [Connerney et al., 1998].

[14] Following the analyses of Leblanc et al. [1994] and Goldstein and Thieman [1981], the observer is assumed to be in the equatorial plane and at an "infinite distance." That is, the position of the source does not change the angle between the observer's line of sight and the magnetic field which only depends on the field direction and the observer's CML.

[15] We then determine the magnetic field pointing vectors along the field line using the VIT4 magnetic field model (Connerney, personal communication). The Goldstein and Thieman [1981] analysis used the field model [Acuña and Ness, 1976] to model the DAM emission. The model is calculated using a spherical harmonic expansion of the field out to fourth order, and then the terms up through the octupole (third order) are kept. The modelled field is constrained by Pioneer 10 measurements. The VIP4 field model [Connerney et al., 1998] uses both Voyager and Pioneer 10 data, along with infra-red (IR) observations of the Io flux tube footprint to calculate the field coefficients to fourth order. The VIT4 field model removes the dependence of the field on Pioneer 10 data. This fourth order model of the internal jovian magnetic field is determined from the location of the IR Io footprint and the theta component of the Voyager magnetic field measurements. Because of the stronger dependence of the higher-order moments on the Io footprint, it is a more accurate model in describing the Io flux tube geometry than the VIP4 model. The location of the Io footprint and the cyclotron frequency at the jovian surface, which limits the highest frequency of emission as a function System III longitude both depend heavily on field model. That dependence is shown in Figures 3 and 4, respectively.

[16] For each Voyager viewing geometry, we compute the System III longitude of Io and then the magnetic field direction along the instantaneous Io flux tube from 1 


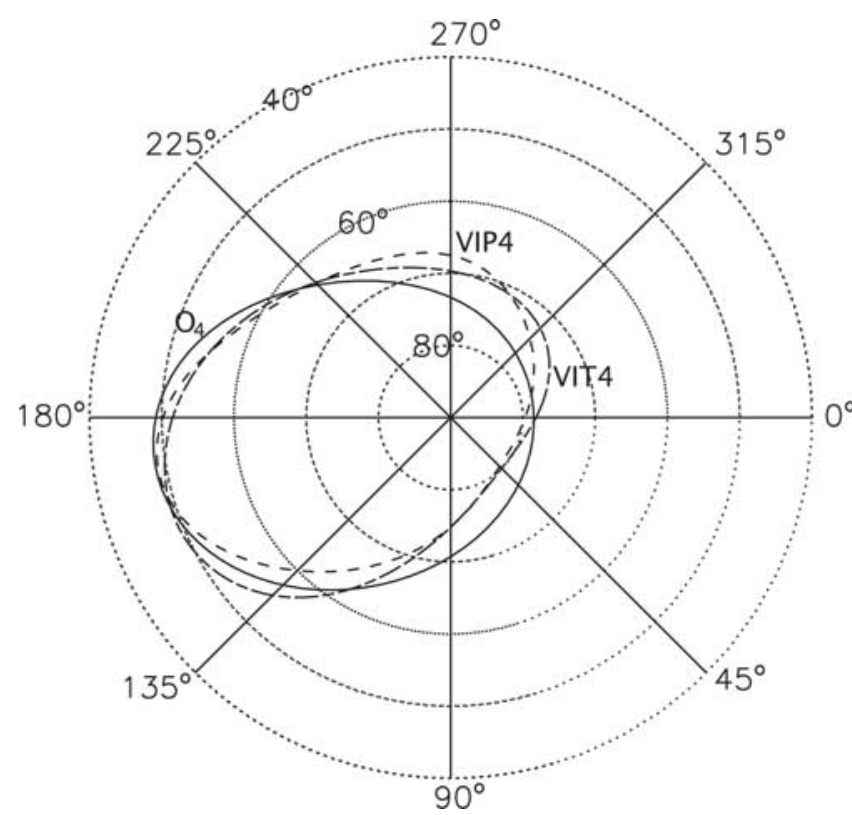

Figure 3. Location of the Io fluxtube footprint in the Northern hemisphere. Field models are labelled on the plot.

$\mathrm{MHz}$ to the maximum cyclotron frequency value as given by Figure 4 at $1 \mathrm{MHz}$ intervals. The emitting field line is fixed in Io's reference frame as in Leblanc et al. [1994] and Queinnec and Zarka [1998]. The angle between the observer's line of sight and the magnetic field is compared to that calculated from a beaming angle function. If these two angles match within $1^{\circ}$, which is the typical width of the hollow cone [Goldstein and Thieman, 1981; Queinnec and Zarka, 1998], the emission is presumed to be observable. Doing this comparison at several frequencies and system geometries permits us to model a dynamic spectrum of the arcs which we then compare with the Voyager observations. A beaming function, $\Theta(f)$, is then adjusted fit the observations. This function is based on the empirical one proposed by Goldstein and Thieman [1981] (Note that we have corrected for the typo in the original publication):

$$
\begin{aligned}
\Theta(f)= & \Theta_{\max } \sin \left[\left(\frac{\pi}{2}\right) \frac{\left(f_{\max }-(1-a) f-a f_{v}\right)}{f_{\max }-f_{v}}\right] \\
& f \geq f_{v} \\
\Theta(f)= & \Theta_{\max } \sin \left[\left(\frac{\pi}{2}\right) \frac{\left((1-a) f+a f_{v}-1\right)}{f_{v}-1}\right] \\
& f \leq f_{v}
\end{aligned}
$$

where $f_{\max }$ is the surface cyclotron frequency of the emitting flux tube and $f_{v}=\left(f_{\max }+1\right) / w$ is the vertex frequency of the arc. The three adjustable parameters are: $a$, which dictates the range of cone angles with frequency (Figure 5a); w, which controls the vertex frequency (Figure 5b); and $\Theta_{\max }$, which sets the maximum beaming angle (Figure 5c). For each source we select 2-3 events with clearly defined arcs in the dynamic spectra from Voyagers 1 and 2 and adjust the above parameters until each arc is simulated in both position and shape.
[17] In their study Goldstein and Thieman [1981] held $\Theta_{\max }=80^{\circ}$ constant and adjusted $a$ and $w$ to find a best fit of $a=.6038$ and $w=3$ for all arcs. However Goldstein and Thieman [1981] did not consider the location of Io in their analysis. The results of our fitting process will be discussed in more detail in section 4.2.

\section{Results}

[18] Table 1 lists the parameters which fit best by inspection for each event and the System III positions of Io and the observer. The beaming angles for the southern hemisphere are measured from the anti-planetward direction (Figure 2c). Our model adequately reproduces the general shape and frequency range of the emission arcs for the $\mathrm{A}, \mathrm{B}$, and $\mathrm{D}$ sources. As we assume that the arc shape is solely a function of beaming angle and Io's location, there is no background emission from non-Io sources. The beaming functions used do not reproduce the shape of the Io-C arcs (Figure 7d), so the beaming angle of these arcs should be investigated by another method.

\subsection{Io-A Source}

[19] The modelled Io-A events are from 13 March 1979 (Figure 7a), 25 March 1979, and 7 July 1979. These vertexlate events have distinct arcs which stand out in the dynamic spectra. They occur at approximately the same source CML, making them easily comparable. Figure 6a displays the beaming angle functions for each event. The shape of the cone angle functions is nearly identical for the three events, with the vertex frequency at $\sim 12 \mathrm{MHz}$, except that the 25 March event requires a larger maximum beaming angle, $\Theta_{\max }$, and the beaming angle function for 7 July spans a larger range of angles. We are able to recreate the three events using emission from the instantaneous Io flux tube, but the modeled arcs reach slightly higher frequency than the observed arcs.

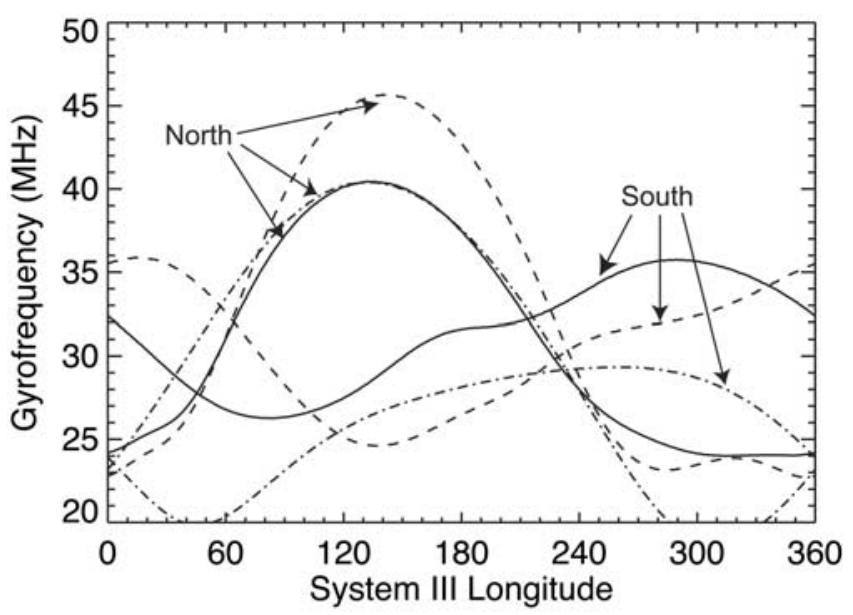

Figure 4. Cyclotron frequency at the 1 bar level for the northern and southern hemispheres using the VIT4 magnetic field model (solid line). The VIP4 surface gyrofrequencies are plotted for comparison (dashed line). Jovian oblateness of $1 / 15.4$ is included. 

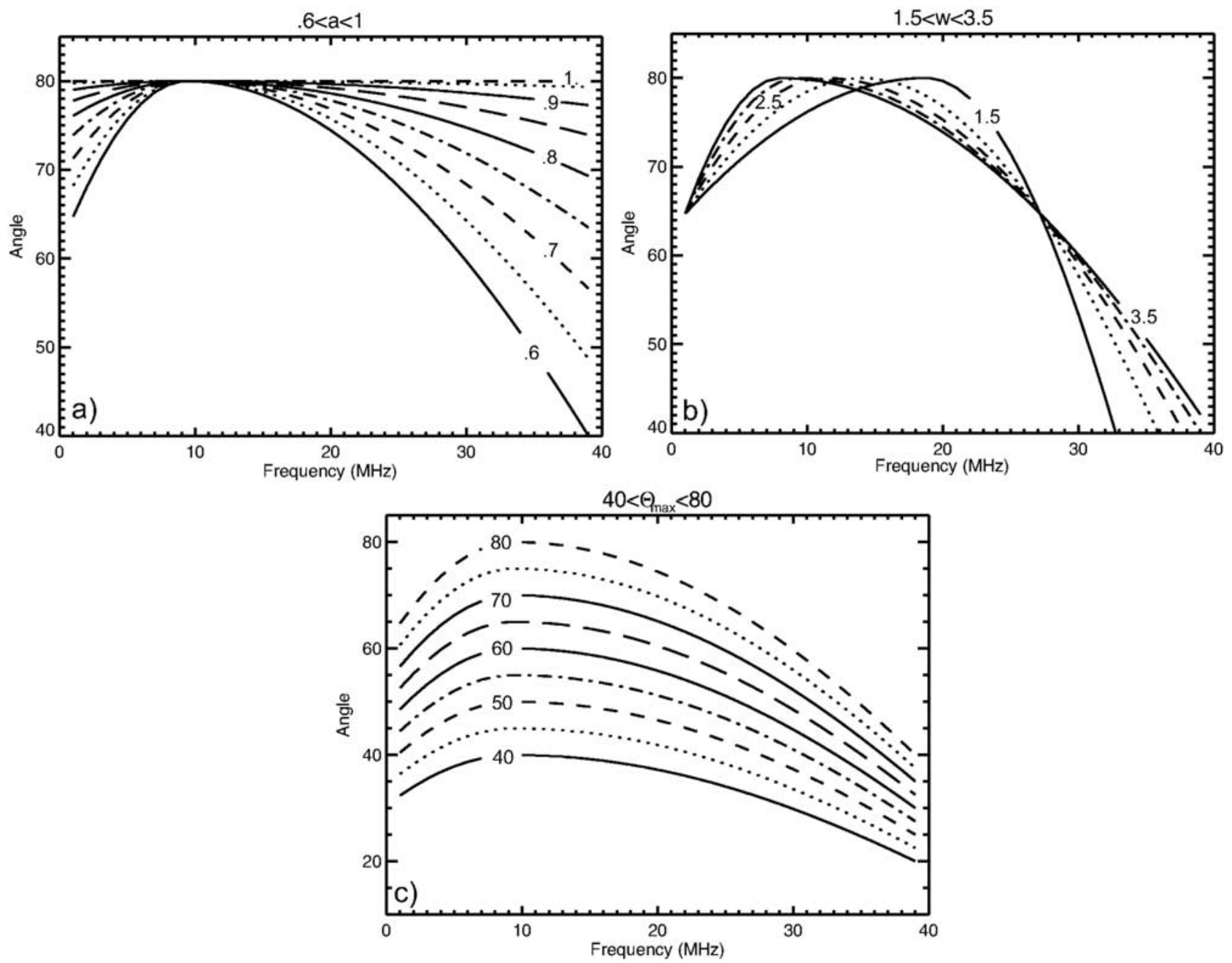

Figure 5. (a) Variation of $\Theta(f)$ with the a parameter. As a increases $\Theta(f)$ flattens out. (b) Variation of $\Theta(f)$ with $w$. The vertex frequency shifts to lower frequencies with increasing $w$. (c) Variation with $\Theta_{\max }$. In addition to directly shifting $\Theta(f)$ in frequency, increasing $\Theta_{\max }$ also affects the range of angles.

Table 1. Beaming Angle Parameters and $\lambda_{I I I}$ of the Observer and Io for Modelled Events

\begin{tabular}{lccccc}
\hline \multicolumn{1}{c}{ Source } & $a$ & $\Theta_{\max }$ & $w$ & $\lambda_{I I I}^{I o}$ & $\lambda_{I I I}^{\text {obs }}$ \\
\hline Sensitivity & $\sim 0.2$ & $\sim 1^{\circ}$ & $\sim 0.5$ & & \\
Io-A & & & & & \\
13 March 1979 & 0.78 & $78^{\circ}$ & 3.0 & $175-190$ & $250-270$ \\
25 March 1979 & 0.79 & $80^{\circ}$ & 3.0 & $170-190$ & $240-270$ \\
7 July 1979 & 0.735 & $78^{\circ}$ & 3.5 & $180-205$ & $250-280$ \\
7 July 1979 & 0.735 & $78^{\circ}$ & 3.5 & $180-205 ; 130-205$ & $250-280 ; 180-280$ \\
Io-B & & & & & \\
21 March 1979 & 0.828 & $92^{\circ}$ & 3.8 & $155-165$ & $70-80$ \\
23 March 1979 & 0.85 & $83^{\circ}$ & 3.8 & $255-265$ & $170-180$ \\
16 July 1979 & 0.85 & $83^{\circ}$ & 3.0 & $235-240$ & $150-160$ \\
23 March 1979 & 0.85 & $83^{\circ}$ & 3.8 & $255-265 ; 230-255$ & $170-180 ; 130-170$ \\
16 July 1979 & 0.85 & $83^{\circ}$ & 3.0 & $235-240 ; 205-235$ & $150-160 ; 110-150$ \\
Io-C & & & & & $300-330$ \\
15 March 1979 & 0.85 & $74^{\circ}$ & 3.0 & $240-265$ & $330-360$ \\
22 March 1979 & 0.85 & $68^{\circ}$ & 3.0 & $270-293$ & $10-70$ \\
Io-D & & & & & $350-50$ \\
7 March 1979 & 0.43 & $70^{\circ}$ & 4.6 & $85-130$ & $10-90$ \\
12 March 1979 & 0.32 & $71^{\circ}$ & 3.2 & $90-95$ & \\
16 March 1979 & 0.34 & $71^{\circ}$ & 3.2 & & \\
\hline
\end{tabular}



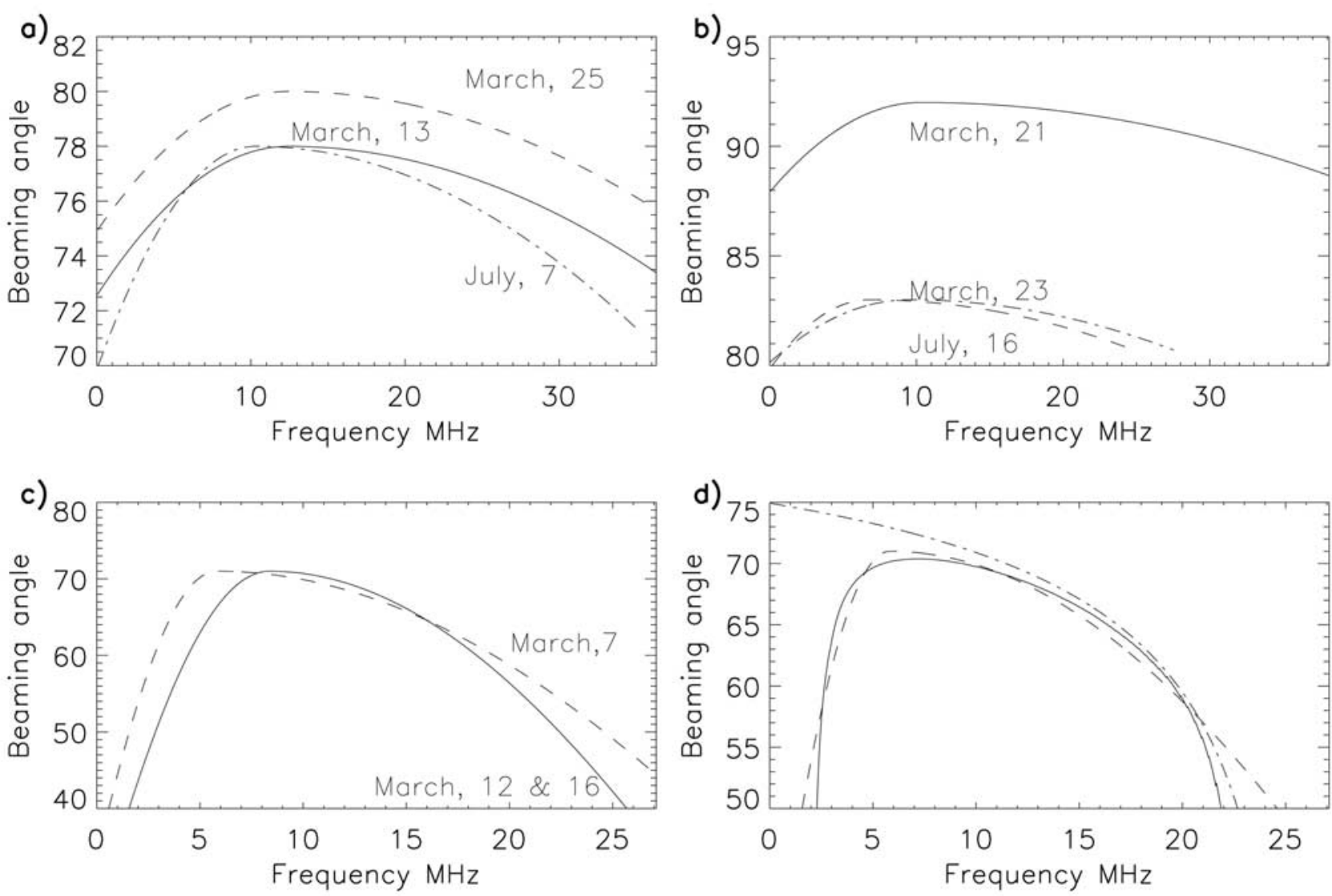

Figure 6. Beaming angle as a function of frequency for the modelled events. (a) Io-A events, (b) Io-B events, (c) Io-D events. (d) Dashed line is the beaming function fit by inspection for the 7 March Io-D event. The continuous line is the beaming function found using CMI theory and including the effect of the refraction inside the source. The dot-dashed line shows the theoretical curve without refraction. We have chosen $v_{0}=v / \sqrt{1-\Omega_{c} / \Omega_{c ; \max }}$ to be consistent with the decrease of beaming angle with frequency.

\subsection{Io-B Source}

[20] The simulated Io-B events are from 21 March 1979, 23 March 1979, and 16 July 1979 (Figure 7b). While the 21 March event is a single distinct arc, the 23 March and 16 July events have multiple arcs which occur over a large range of observer CML, implying that the emission is due to both the instantaneous Io flux tube (for the main arc) and wake (for secondary arcs). We model the shape of the wake arcs using the same beaming angle function, but with emitting field lines located downstream of Io in the wake and compare to the secondary arcs. As our model does not reproduce the arc shapes for all of the wake emissions (Figure $7 \mathrm{~b}$ ), the parameters of the beaming function must vary for each arc. The main arcs are well fit except at high frequencies where the emission appears as a trailing tail. Queinnec and Zarka [1998] showed that at these frequencies the beaming angle decreases abruptly, which is not compatible with the chosen beaming angle function, $\Theta(f)$ (equation (1)). Moreover, to be consistent with the limitation at high frequencies because of the surface cyclotron frequency, these emissions must be emitted from field lines connected to the wake.

[21] The 21 March event is considerably different from the two other events both in longitude range and in beaming angle. Its beaming angle function, $\Theta(f)$, reaches angles greater than $90^{\circ}$ and its emission appears left-handed, consistent with CMI theory, which predicts that the RH emission cannot be propagated at obtuse angles. Hence this event may result from a different process than the typical Io-B events.

\subsection{Io-D Source}

[22] The modelled Io-D events are 7 March 1979 (Figure 7c), 12 March 1979, and 16 March 1979. All three events have a single arc which spans a broad range of observer CML. Figure $6 \mathrm{c}$ shows the beaming angle functions for each event. The angular range of $\Theta(f)$ is large; $\sim 40^{\circ}$ as opposed to $5^{\circ}$ for the A events which may be because of the fact that the model fits at high frequencies near the surface cyclotron frequency, where a strong decrease of the beaming angle is expected [Queinnec and Zarka, 1998; Lecacheux et al., 1998].

[23] The $\mathrm{D}$ events are ideal in that the same parameters for the beaming angle function, $\Theta(f)$, are applied to multiple events (12 March and 16 March), suggesting a general shape of the Io-D arcs. It is important to note that these two events occur over the same range of longitude. The modelled 7 March arc follows the observed arc for nearly all frequencies; and the 12 March and 16 March modelled arcs match except at low frequencies. Each 

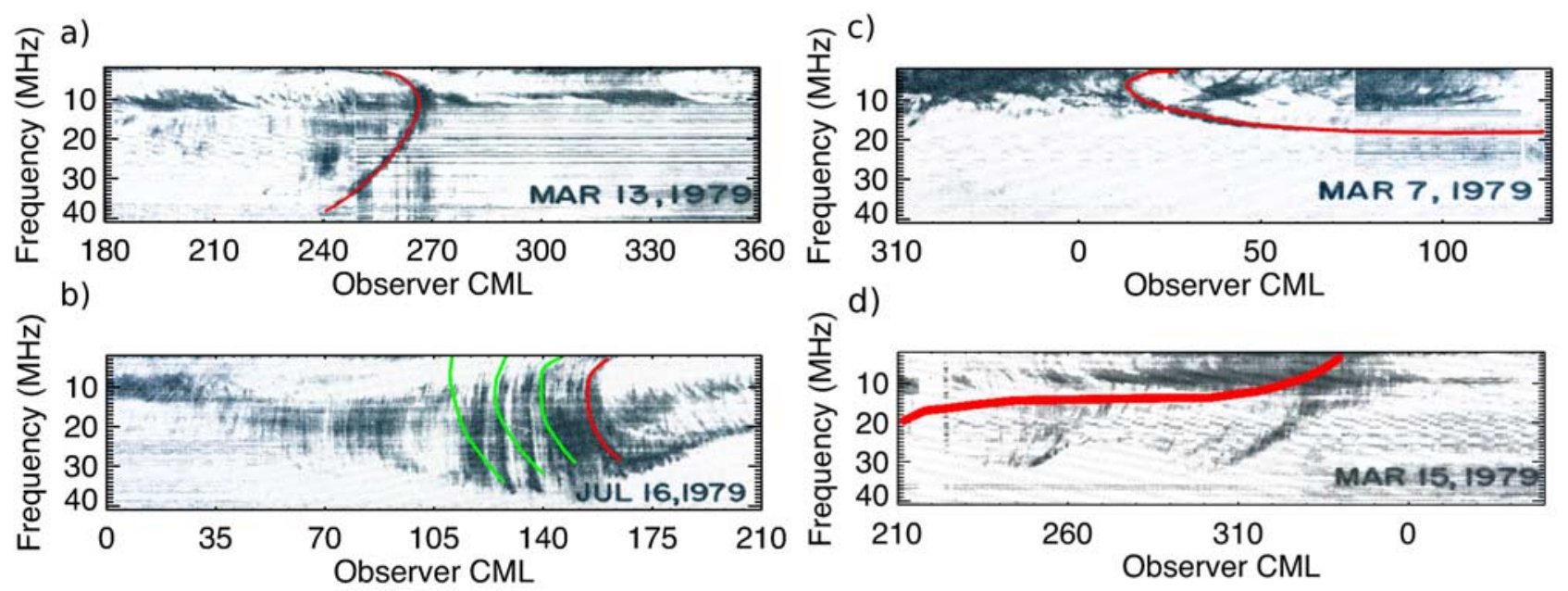

Figure 7. The modelled arcs (red curves) are overplotted on the observed ones: (a) A event, (b) B event, (c) D event. The green curves on the (b) figure show the secondary arcs modeled assuming a wake emissions and the same beaming angle function as for the main arc. Although the position of the secondary arcs are consistent with wake emissions, the beaming angle function must be different than those of the main arc. (d) Modelled C-event using the instantaneous Io flux tube. Simulated arc does not match observation.

modelled arc is due to emission from the instantaneous Io flux tube.

\section{Discussion}

\subsection{Comparison Between the Sources}

[24] Although the shape of the A and B arcs, both righthanded emission, are different, the parameters used for the beaming angle function are similar, with a vertex frequency between $10 \mathrm{MHz}$ and $12 \mathrm{MHz}$ and a beaming angle with a mean value around $80^{\circ} \pm 2^{\circ}$ which varies slowly over a limited range. The exception is the Io-B tail which is not fit by this analysis. These two sources are generally composed of a main arc and secondary arcs, that may be due to emitting field lines connected to the wake. It is difficult to determine anything definitive about the relationship between the $\mathrm{C}$ and $\mathrm{D}$ events as we were unable to reproduce the $\mathrm{C}$ event arcs with our chosen. Therefore, the beaming angle function is different between the two source locations, with the exception that both have a maximum beaming angle of $70^{\circ}$.

[25] Except for the difference in the maximum emission frequency of the arcs, which is due the asymmetry of the jovian magnetic field, the main difference between the northern and southern emissions is the difference of beaming angle. The northern hemisphere emissions have a $\Theta(f)$ which ranges from $70^{\circ}$ to $90^{\circ}$ while the beaming angle for the southern hemisphere emissions ranges from $50^{\circ}$ to $70^{\circ}$. This may reflect a strong difference between emission processes in each hemisphere, or a different lead angle between the Io field line and the emitting field line. Moreover, the wake emissions occur more often with northern sources.

\subsection{Comparison With Past Work}

[26] The most important difference between our model and that of Goldstein and Thieman [1981] is that the emitting field line in our model is fixed in Io's reference frame, which is consistent with an emission due to the IoJupiter interaction. Goldstein and Thieman [1981] excited the magnetic field lines every $20^{\circ}$ and hence the 'best-fit' parameters that they found are independent of any motion through the system as well as independent of Io's location. We also allow for a beaming angle function which may differ for each source in our model, whereas it is fixed for all the sources in their model. Goldstein and Thieman [1981] assumed the same function for all sources but did allow for a different vertex frequency, the frequency at which the beaming angle function switches from increasing to decreasing, between the early and late sources. We vary the parameters between sources and also adjust the maximum beaming angle. Therefore, this analysis investigates broader variations $\Theta(f)$ in between the sources. In particular, we find that the mean value of the beaming angle is different between the northern and southern hemispheres.

[27] Another difference between our analysis and that of Goldstein and Thieman [1981] is due to the use of the VIT4 field model instead of the $\mathrm{O}_{4}$ field model. The VIT4 field model has a higher-surface gyrofrequency than the $\mathrm{O}_{4}$ field model, particularly for the southern hemisphere (Figure 4). Hence our modelled arcs extend to higher frequencies. In addition, the higher-order moments included in the VIT4 field model adjust the field geometry at high latitudes, slightly shifting the shapes of the arcs at high frequencies. The direction of the shift is dependent on the longitude of the $\lambda_{I I I}$ emission.

[28] Our modelled beaming angles for the 13 March and 7 July 'A' arcs are consistent with those derived by Menietti et al. [1984]. The Menietti et al. [1984] analysis modelled each arc using two assumed Doppler shift values. Our modelled beaming angles are less than the high-Doppler shifted source results for low frequencies, but are larger at middle and high frequencies $(f \geq 8 \mathrm{MHz})$. As we do not take Doppler shift into account in our model, this is likely do to the magnetic field model used. Our modelled arcs 

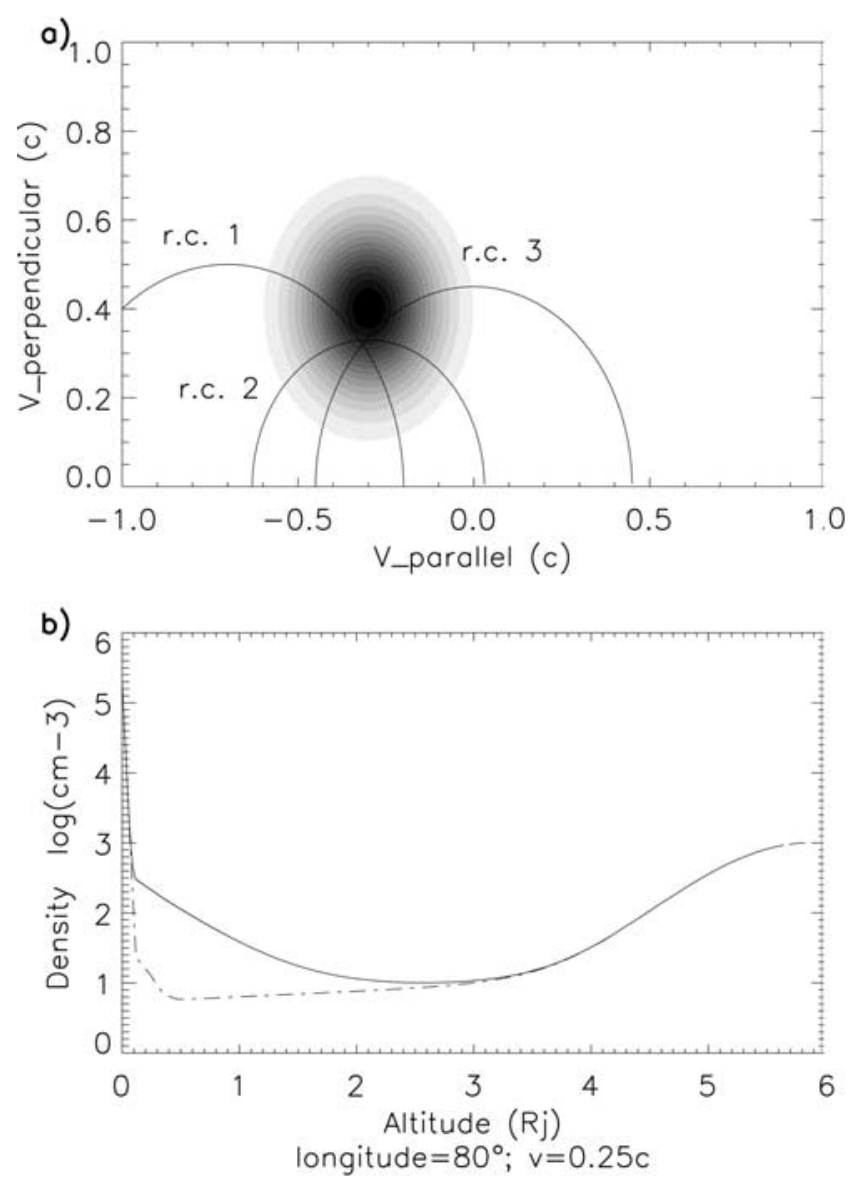

Figure 8. (a) Example of a ring distribution unstable relative to CMI. This distribution is unstable relative to several resonance conditions. The resonance circle r.c. 1 center is given by $\left(v_{0}=v / \cos \alpha\right)$, the r.c. 2 center by $v_{0}=$ $v \cos \alpha$ and the r.c. 3 center by $v_{0}=0$. (b) Density profile along the emitting field line used to fit the data (Figure 6d). The altitude is given relative to jovian surface. The dashed line corresponds to the model of $\mathrm{Su}$ et al. [2003] and the continuous line to the model we use to fit the beaming angle. In our model, a minor species with a temperature of $\sim 4 \mathrm{eV}$ is added to fit the vertex frequency.

extend to higher frequencies, which is attributed to our calculation of the gyrofrequency at $1 \mathrm{MHz}$ intervals down to the surface while they chose a set number of emission frequencies for their analysis.

[29] Queinnec and Zarka [1998] derive the beaming angle function by working backward from the system geometry. The beaming angle function, $\Theta(f)$, is determined for both the instantaneous Io flux tube and multiple lag angles (wake emission locations downstream of Io). They find an average beaming angle of $70^{\circ}-75^{\circ}$. Moreover their measures show a decrease of the beaming angle at the lowest and the highest frequencies for all the observed arcs. For all sources our results are consistent with those obtained by Queinnec and Zarka [1998] for a lead angle of approximately $20^{\circ}$. They observe comparable maximum beaming angles, vertex frequencies, and variations of the beaming angle in the frequency ranges common to both studies (4 $\mathrm{MHz}-20 \mathrm{MHz}$ for the southern and $8 \mathrm{MHz}-30 \mathrm{MHz}$ for the northern). At higher frequencies Queinnec and Zarka [1998] observe an abrupt decrease in the beaming angle for emission, particularly for the B and $\mathrm{C}$ sources. The main difference between the two studies is that Queinnec and Zarka [1998] directly measure the beaming angle whereas we suppose an empirical beaming angle function to obtain the shape of the emission. Thus their measurements may be more precise, but they cannot tell if the beaming angle function they obtained uniquely results in the observed emissions or if it should generate other emissions that are not observed. Our model shows that the observed arcs are the only visible emissions for our beaming angle function.

\subsection{Physical Explanation}

[30] The beaming angle function we use is a strictly empirical one derived by Goldstein and Thieman [1981] from the Voyager observations. Moreover, it does not describe the abrupt variations of the beaming angle at high frequencies (near the cyclotron frequency at the surface) [Queinnec and Zarka, 1998]. Therefore we need a beaming angle function, derived from a physical theory, which is consistent with equation (1) far from the jovian ionosphere and decreases abruptly near it. Such a function can be deduced from the CMI theory if we make reasonable assumptions about the density profile along the field line and some properties of the emitting particles. For a more in depth review of maser emission, the reader is referred to more detailed discussions [ $\mathrm{Wu}$ and Lee, 1979; Melrose and Dulk, 1982; Winglee and Dulk, 1986; Ergun et al., 2000; Pritchett et al., 2002].

[31] The CMI is a resonant interaction between electrons with a velocity, $v$, and a circularly polarized wave with a frequency, $f$. The resonance condition is given by:

$$
f=f_{c} / \gamma+\frac{k_{\|} v_{\|}}{2 \pi}
$$

where the $\|$ subscript refers to the direction parallel to the magnetic field lines and $f_{c}$ is the electron cyclotron frequency. This equation describes a sphere in the velocity space, whose center $v_{0}$, located on the $v_{\|}$axis, is given by:

$$
v_{0}=\frac{k_{\|} c^{2}}{2 \pi f_{c}}=\frac{f_{c} N}{f_{c}} \frac{\mathbf{k} \cdot \mathbf{b}}{k}=\frac{f_{c} N}{f_{c}} \cos \Theta
$$

where $N$ is the refraction index given by the AppeltonHartree dispersion relation in the cold plasma approximation. The beaming angle function, $\Theta(f)$, depends on the resonance sphere and on the refraction index, which in turn depends on the plasma frequency. Finally, the determination of the resonance sphere depends on the electron distribution, $F_{e}$. The emission is produced on the $\mathrm{R}-\mathrm{X}$ mode which implies that the allowed solutions to the resonance condition (equation (2)), which occur along the resonance sphere, must correspond to frequencies above the $\mathrm{R}-\mathrm{X}$ cutoff frequency. The resonance sphere for which the surface integral of $v_{\perp}^{2}$ $\frac{d F_{e}}{d v_{\perp}}$ is maximum [Wu and Lee, 1979] yields the most amplified, and hence observed, mode. The most common distributions unstable relative to $\mathrm{CMI}$ in the auroral zone are the loss-cone, the ring and the shell distributions [Ergun et $a l ., 2000,2006]$. It results in three main relations between 
the resonance sphere center, $v_{0}$, and the local cyclotron frequency, $f_{c}$, depending on the velocities for which the gradient $\frac{d F_{e}}{d v_{\perp}}$ is positive (with $\alpha$ the resonant particle pitchangle, taken to be equal to the loss-cone aperture arccos $\left(\sqrt{1-f_{c} / f_{c ; \max }}\right)$ where $f_{c ; \text { max }}$ is the cyclotron frequency at the jovian surface):

$$
v_{0}=v / \cos \alpha=v / \sqrt{1-f_{c} / f_{c ; \max }}
$$

predominant for a loss-cone distribution. The resonance sphere is tangent to the loss-cone.

$$
v_{0}=v_{\|}=v \cos \alpha=v \sqrt{1-f_{c} / f_{c ; \max }}
$$

predominant for a ring distribution. The ring is a great circle of the resonance sphere.

$$
v_{0}=0
$$

predominant for a shell distribution. The resonance sphere is tangent to the inner edge of the shell.

[32] Because of the symmetries, the velocity space can be reduced to the $\left(v_{\|}, v_{\perp}\right)$ plane. Therein the resonance condition is represented by a resonance circle, whose center is the same as that of the resonance sphere $\left(v_{0}\right)$. The resonance circles corresponding to the above equations are shown in Figure 8 in the case of a ring distribution (this distribution is unstable relative to these three instabilities). The first instability (equation (4)) is the only one which presents an increase of $v_{0}$, and thus a decrease of the beaming angle $\Theta \propto \arccos v_{0}$ (equation (3)), when the local cyclotron frequency $f_{c}$ increases and particularly when it reaches the surface cyclotron frequency $f_{c \text {;max }}$. Since this decrease of the beaming angle $\Theta$ is seen in all the beaming angle studies (including ours), we assume a "loss-cone like" emission. Thus from equations (2), (3), and (4) the wave parameters are, in the weak relativistic approximation:

$$
\begin{gathered}
k_{\|}=\frac{2 \pi f_{c} v_{0}}{c^{2}}=\frac{2 \pi f_{c} v}{c^{2} \cos \alpha} \\
f \simeq f_{c}\left(1-\frac{v^{2}}{2 c^{2}}\right)+\frac{k_{\|} v_{\|}}{2 \pi}=f_{c}\left(1-\frac{v^{2}}{2 c^{2}}\right) \\
+\frac{f_{c} v}{c^{2} \cos \alpha} v \cos \alpha=f_{c}\left(1+\frac{v^{2}}{2 c^{2}}\right) \\
\Theta=\arccos \left(\frac{f_{c} v}{f N c \cos \alpha}\right)
\end{gathered}
$$

This assumption is consistent with a loss-cone distribution, but also describes oblique emission from other auroral distributions. Such a distribution is invoked to explain Iocontrolled DAM bursts with millisecond time-scale [Hess et al., 2007a]. Moreover in this case the beaming may be obtained using the cold plasma approximation. Figure $6 \mathrm{~d}$ shows a simulation of the theoretical beaming angle function for the 7 March event. The dot-dashed curve shows the theoretical beaming angle function $\Theta(f)$ for a "loss-cone like" beaming without any density effects $(N=1)$. It shows an abrupt decrease of the beaming angle at high frequencies, consistent with the observations of Queinnec and Zarka [1998]; Lecacheux et al. [1998], but it is not consistent with the beaming angle function, inferred from Goldstein and Thieman [1981] and our study (dot-dashed lines). We then assume the density profile shown by the continuous curve in Figure $8 \mathrm{~b}$. The result is shown in Figure $6 \mathrm{~d}$ by the continuous curve and is fully consistent with the observations (dashed curve). The density model is consistent with the model of $\mathrm{Su}$ et al. [2003] (dashed curve in Figure 8b), but without an auroral cavity and with a minor $(0.1 \%$ in density) ionospheric hydrogen population with a temperature about $4 \mathrm{eV}$.

[33] As we do not directly fit the data, but instead apply an empirical model which is then fit to the data, this model may be not representative of the density in the Io flux tube. Further work should be done, with a direct fit of the data, to obtain a more realistic density profile.

[34] The resonant particle energy is $\sim 16 \mathrm{keV}$ in this model, in order to obtain a maximum beaming angle of $70^{\circ}$. Northern sources, whose maximum beaming angle is about $80^{\circ}$, involve particles with $\sim 5 \mathrm{keV}$, consistent with the measurements made by Zarka et al. [1996]; Hess et al. [2007b] for the millisecond Io-controlled bursts.

\section{Conclusions}

[35] The purpose of this study was to model the decametric arcs observed by the Voyager 1 and 2 spacecraft by modifying the beaming angle function, $\Theta(f)$. To do this we created a model which determined the magnetic field vector at each emission frequency and then calculated the angle between the emission point and the observer. A beaming angle function was then applied and adjusted until the modelled arcs matched the Voyager observations. We then looked at the four source regions separately and compared the results within each source region; for early and late arcs, Northern and Southern hemisphere emission, instantaneous Io flux tube and wake emission, and finally with past studies. The main results are as follows:

[36] 1. The functional form of $\Theta(f)$ (equation (1)) derived in Goldstein and Thieman [1981] is satisfactory for modelling arcs in the Io-stationary reference frame (except at the frequencies near the Jovian surface), provided the maximum cone angle is allowed to vary. Although we were unable to reproduce the $\mathrm{C}$ arcs, reasonable results were attained for the $\mathrm{A}, \mathrm{B}$, and $\mathrm{D}$ source regions.

[37] 2. As the model assumes emission solely along the instantaneous Io flux tube, or at fixed field lines within the wake, the jovian arc shape is due ONLY to the beaming angle of the emissions along an unique magnetic field line fixed in Io's reference frame. Variations in the shape and frequency are due to differences in the magnetic field structure in System III.

[38] 3. From the measurements we made and those of previous studies, we propose that the beaming angle function may be simply deduced from the CMI theory. It may depend only on the density profile and energy of the emitting electrons. Thus further modelling, using a theoret- 
ical profile of the beaming angle instead of an empirical one, may permit one to get an estimation of the electron energy and of its variations with longitude and hemisphere.

[39] 4. The beaming angle function and the shape of the arcs are similar within each source region, even if the maximum of the beaming angle $\Theta_{\max }$ may vary. According to the theoretical interpretation of the beaming angle function proposed in this paper, this variation may correspond to different energies of the emitting particles.

[40] 5. The beaming angle function varies with hemisphere. For the early sources, the angular range of the emission is narrower in the North $\left(\sim 10^{\circ}\right.$ for the B source versus $\sim 35^{\circ}$ for the $\left.\mathrm{D}\right)$. This may correspond to different energies of the emitting particles in the northern and southern hemispheres.

[41] 6. Wake emission is necessary to model the secondary arcs of the B and A events. The beaming angle function, $\Theta(f)$, must change downstream of Io as the wake arcs span a smaller range in frequency and observer CML. Preliminary investigation implies a more constant beaming angle function for wake emission.

[42] Acknowledgments. Wolfgang Baumjohann thanks Michael Kaiser and John Menietti for their assistance in evaluating this paper.

\section{References}

Acuña, M. H., and N. F. Ness (1976), Results from the GSFC Fluxgate Magnetometer on Pioneer 11, in Jupiter, pp. 830-847, University of Arizona Press.

Bigg, E. K. (1964), Influence of the Satellite Io on Jupiter's Decametric Emission, Nature, 203, 1008-1010.

Boischot, A., A. Lecacheux, M. L. Kaiser, M. D. Desch, J. K. Alexander, and J. W. Warwick (1981), Radio Jupiter after Voyager-An overview of the planetary radio astronomy observations, J. Geophys. Res., 86, 82138226.

Burke, B. F., and K. L. Franklin (1955), Observations of a variable radio source associated with the planet Jupiter, J. Geophys. Res., 60, 213-217.

Clarke, J. T., D. Grodent, S. W. H. Cowley, E. J. Bunce, P. Zarka, J. E. P. Connerney, and T. Satoh (2004), Jupiter's aurora, in Jupiter. The Planet, Satellites and Magnetosphere, pp. 639-670, Cambridge University Press.

Clarke, J. T., et al. (1998), Hubble Space Telescope imaging of Jupiter's UV aurora during the Galileo orbiter mission, J. Geophys. Res., 103, 20,21720,236 .

Connerney, J. E. P., M. H. Acuña, N. F. Ness, and T. Satoh (1998), New models of Jupiter's magnetic field constrained by the Io flux tube footprint, J. Geophys. Res., 103, 11,929-11,939.

Ergun, R. E., C. W. Carlson, J. P. McFadden, G. T. Delory, R. J. Strangeway, and P. L. Pritchett (2000), Electron-cyclotron maser driven by chargedparticle acceleration from magnetic field-aligned electric fields, Astrophys. J., 538, 456-466.

Ergun, R. E., Y.-J. Su, L. Andersson, F. Bagenal, P. A. Delemere, R. L. Lysak, and R. J. Strangeway (2006), S bursts and the Jupiter ionospheric
Alfvén resonator, J. Geophys. Res., 111, A06212, doi:10.1029/ 2005JA011253.

Goldstein, M. L., and J. R. Thieman (1981), The formation of arcs in the dynamic spectra of Jovian decameter bursts, J. Geophys. Res., 86, 85698578

Hess, S., F. Mottez, and P. Zarka (2007a), Jovian S-bursts generation by Alfvén waves, J. Geophys. Res., 112, A11212, doi:10.1029/2006JA012191.

Hess, S., P. Zarka, and F. Mottez (2007b), Io-Jupiter interaction, millisecond bursts and field-aligned potentials, Planet. Space Sci., 55, 89-99, doi:10.1016/j.pss.2006.05.016.

Leblanc, Y., G. A. Dulk, and F. Bagenal (1994), On Io's excitation and the origin of Jupiter's decametric radiation, Astron. Astrophys., 290, 660673.

Lecacheux, A., M. Y. Boudjada, H. O. Rucker, J. L. Bougeret, R. Manning, and M. L. Kaiser (1998), Jovian decameter emissions observed by the Wind/WAVES radioastronomy experiment, Astron. Astrophys., 329, $776-784$.

Melrose, D. B., and G. A. Dulk (1982), Electron-cyclotron masers as the source of certain solar and stellar radio bursts, Astrophys. J., 259, 844858, doi:10.1086/160219.

Menietti, J. D., J. L. Green, S. Gulkis, and N. F. Six (1984), Jovian decametric arcs-An estimate of the required wave normal angles from threedimensional ray tracing, J. Geophys. Res., 89, 9089-9094.

Pritchett, P. L., R. J. Strangeway, R. E. Ergun, and C. W. Carlson (2002), Generation and propagation of cyclotron maser emissions in the finite auroral kilometric radiation source cavity, J. Geophys. Res., 107(A12), 1437, doi:10.1029/2002JA009403.

Queinnec, J., and P. Zarka (1998), Io-controlled decameter arcs and IoJupiter interaction, J. Geophys. Res., 103, 26,649-26,666.

Saur, J., F. M. Neubauer, J. E. P. Connerney, P. Zarka, and M. G. Kivelson (2004), Plasma interaction of Io with its plasma torus, in Jupiter. The Planet, Satellites and Magnetosphere, pp. 537-560, Cambridge University Press.

Su, Y.-J., R. E. Ergun, F. Bagenal, and P. A. Delamere (2003), Io-related Jovian auroral arcs: Modeling parallel electric fields, J. Geophys. Res., 108(A2), 1094, doi:10.1029/2002JA009247.

Thomas, N., F. Bagenal, T. W. Hill, and J. K. Wilson (2004), The Io neutral clouds and plasmatorus, in Jupiter. The Planet, Satellites and Magnetosphere, pp. 561-591, Cambridge University Press.

Willes, A. J., D. B. Melrose, and P. A. Robinson (1994), Elliptically polarized Jovian decametric radiation: An investigation of the electron cyclotron maser mechanism, J. Geophys. Res., 99, 21,203-21,211.

Winglee, R. M., and G. A. Dulk (1986), The electron-cyclotron maser instability as a source of plasma radiation, Astrophys. J., 307, $808-$ 819, doi: $10.1086 / 164467$

Wu, C. S., and L. C. Lee (1979), A theory of the terrestrial kilometric radiation, Astrophys. J., 230, 621-626, doi:10.1086/157120.

Zarka, P. (1998), Auroral radio emissions at the outer planets: Observations and theories, J. Geophys. Res., 103, 20,159-20,194.

Zarka, P. (2000), Radio Emissions from the Planets and their Moons, in Radio Astronomy at Long Wavelengths, edited by R. G. Stone et al., pp. 167-178, AGU.

Zarka, P., T. Farges, B. P. Ryabov, M. Abada-Simon, and L. Denis (1996), A scenario for Jovian S-bursts, Geophys. Res. Lett., 23, 125-128.

S. Hess and L. C. Ray, Laboratory for Atmospheric and Space Physics, University of Colorado, UCB392, Boulder, CO 80309-0392, USA. (1ray@colorado.edu) 